\title{
Morphological and allozyme studies of small terrestrial snails (Opeas sp., Subulina sp., and Huttonella bicolor) collected from Peninsular Malaysia
}

\begin{abstract}
Shell morphological characters and allozyme electrophoresis were used to study the relationships among six geographical populations of land snails collected from Peninsular Malaysia. Allozyme electrophoresis was used to study the genetic variations to complement the morphological features studied that included shell lengths, numbers of whorls and shell colour. Ten loci coding for six enzymes (MDH, LAP, ALP, PGM, G6PDH, and EST) could be reliably scored in samples from the six populations studied. The dendrogram showed two major clusters with one cluster comprising Subulinidae populations from Perak, Selangor, Johor, Terengganu, and Pahang while the other cluster included only the Streptaxidae Huttonella bicolor (red) population. The Subulinidae populations were grouped into two subclusters: one subcluster included the Subulina sp. populations from Perak, Selangor, and Johor, while the other subcluster included the Opeas sp. populations from Terengganu and Pahang. Morphological features can identify the different families ant therefore they can complement the allozyme genetic studies on the land snail populations. Like other reports in the literature, our results also underline the importance of a genetic approach in conjunction with a morphological approach, for discriminating land snail species. The present results suggest that small land snails, which were similar in colour but different in sizes, were not of the same family/genus.
\end{abstract}

